\title{
Pattern and Ganzfeld electroretinograms in macular disease
}

\author{
G. B. ARDEN, R. M. CARTER, AND A. MACFARLAN
}

From the Electrodiagnostic Clinic, Moorfields Eye Hospital, and the Department of Clinical Ophthalmology, Institute of Ophthalmology, City Road, London ECIV 2PD

SUMMARY The pattern electroretinogram has been found to be abnormal in all except three cases of maculopathy. This is in contrast to other electrophysiological tests, which are frequently unaffected. The degree of reduction is related to the loss of visual acuity. The response to focal flashes is less affected than the response to pattern.

The electroretinogram (ERG) has not proved to be of significance to the study and diagnosis of macular degenerations. This is partly because the ERG tests the function of large retinal areas, while the macular lesion occupies a small fraction of the retina. In addition the ERG is dominated by scotopic responses, while the macular contains many cones. Attempts have been made to elicit the photopic ERG of the macular region. Although the responses to focal flashes have been recorded under clinical conditions, ${ }^{1-3}$ there are considerable difficulties in ensuring that they originate from a defined retinal area. Another technique is to use a reversing pattern ${ }^{4}$ as a stimulus, since stray light in the retinal zones outside the image of the pattern remains constant as the pattern changes. Recently it has been shown that the pattern ERG (PERG) can be used as a clinical tool in the investigation of retinal disease. . $^{5-9}$

The object of this paper is to give normative PERG data and to describe the findings in defined forms of macular degeneration. It has been claimed that the PERG, although similar in waveform to the response evoked by flashes, may be produced by a different mechanism. Therefore we have recorded the ERGs to focal and diffuse illumination in such patients, to determine whether the results support the supposition that the PERG is abnormal under conditions where the same retinal area can produce a normal flash response.

\section{Material and methods}

Ganzfeld ERGs were recorded as previously described. "In brief, earth and reference electrodes

Correspondence to Professor G. B. Arden, Institute of Ophthalmology, Judd Street, London WC1H 9QS. were placed on the forehead. The corneal electrode ${ }^{5}$ was placed in the lower fornix. Graded intensities of blue light were used to evoke rod ERGs, and red light was used to evoke separable cone and rod ERGs. Cone ERGs were also evoked by flickering white light. Intense white light was used to evoke oscillatory potentials. The amplitude and time to peak of the major waves were measured for every stimulus condition. The light source was a stroboscope (Grass PS22) which had been recently calibrated. ${ }^{\prime \prime \prime}$

Grading of the ERG. In common with other workers we find that there is a large normal variation in the Ganzfeld ERG. When a relatively homogeneous group of patients is investigated, the average values of the group may be compared with those of a control group. To facilitate comparison of an individual's results to the control values we have normalised the results by subtracting the mean normal value for any condition from the individual's result, and dividing the result by the mean. Thus, if the ERG was half sized, the result obtained would be $-0 \cdot 5$. In this way we can compare the results obtained for a number of conditions. Since there are 22 observations in a full ERG protocol, and there are reasons for assuming that they are independent, the method of calculation described above permits a better statistical treatment of the results, and allows us to determine whether the average of results obtained from a patient differs significantly from the normal.

Pattern ERGs (PERG) were recorded as previously described. ${ }^{56} " 1$ The stimulator ${ }^{12}$ generated a reversing pattern on a TV monitor. The screen displayed checkerboards of squares which subtended $1^{\circ}, 0 \cdot 5^{\circ}$, or $8^{\circ}$ at the eye. The last was used to record focal luminance responses (see below). In the first experi- 
ments a monitor was employed which gave an $84 \%$ contrast with a mean luminance of $50 \mathrm{~cd} \cdot \mathrm{m}^{-2}$, and the pattern subtended $18 \times 22^{\circ}$. Stimulation frequencies were 2 or $3 \mathrm{~Hz}$-i.e., 4 or 6 reversals $\mathrm{s}^{-1}$ Lighting conditions for this part of the investigation were mesopic. A second monitor was also used which produced checkerboards of $98 \%$ contrast at a mean luminance of $140 \mathrm{~cd} . \mathrm{m}^{-2}$ The pattern subtended $20 \times 15^{\circ}$ (in a few experiments $15 \times 15^{\circ}$ ). The stimulation frequency was 6 reversals $\mathrm{s}^{-1}$ The monitor was used with a large white mask illuminated to $100 \mathrm{~cm} \cdot \mathrm{m}^{-1}$ to ensure that the patient was thoroughly lightadapted. The PERGs evoked by this second monitor were significantly larger than for the first despite the smaller size and the bright surround. The television frame rate was given by the frequency of the mains $(50 \mathrm{~Hz})$. The stimulus repetition rate was made independent of this frequency to avoid $50 \mathrm{~Hz}$ interference. Each record consists of four or more summations of 200-500 individual responses. A small light-emitting diode (LED) placed centrally on the screen served as a fixation spot. Some patients who had central scotomata were unable to see the central fixation target. In such cases the LED was positioned on the screen in such a way that the visual axis met the screen centre, and the patient could see the LED with the preferred part of the peripheral retina.

Electroculograms (EOG) were recorded as previously described. ${ }^{13}$

Selection of patients. All the patients had been referred from the Retinal Diagnostic Department, where the examination included fundus photography and (in most cases) fluorescein angiography. Visual fields and visual acuity were measured. In 14 cases the patients had already been specially investigated and had classical Stargardt's disease; they formed a homogeneous subgroup. They had well demarcated 'punched out' retinal pigment epithelium (RPE) atrophy at the macula, so choroidal vasculature was easily visible. More peripherally the changes were discontinuous with a pattern of pigment clumping and RPE atrophy. These changes fell
Table 1 Diagnoses of the patients examined

\begin{tabular}{ll}
\hline $\begin{array}{l}\text { Number of } \\
\text { patients }\end{array}$ & Condition \\
\hline 14 & Stargardt's discase \\
2 & Central serous retinopathy \\
3 & Bull's cye maculopathy \\
2 & Vitelliruptive macular \\
degencration \\
2 & X-linked retinoschisis \\
1 & Juvenile maculopathy \\
12 & Gencral maculopathy \\
3 & General retinopathy \\
1 & Functional \\
\hline
\end{tabular}

short of the disc. The RPE contained 'flecks,' which angiography showed as opaque accumulations obscuring the choroidal vascular fluorescence. In cases of familial disease affected relatives of the propositus were also examined. The remainder fell into various categories (Table 1).

\section{Results}

\section{PSYCHOPHYSICS}

Visual acuity in these patients ranged from $6 / 5$ to counting fingers at 1 metre (CF). Average vision for those better than $C F$ was between $6 / 24$ and $6 / 36$ (acuity of 5 minutes arc).

Colour vision. The subset of patients with Stargardt's disease selected for further investigation by extended pattern and focal ERGs tests also had their colour vision examined by the use of the HRR (Hardy, Rand, Rittler) pseudoisochromatic plates. A mild red green (protan) defect was found in 11 patients, and two failed to identify the patterns. The remaining patient had normal colour perception (visual acuity better than 6/5).

\section{ELECTROPHYSIOLOGY}

Many patients in this series were referred to the Electrodiagnostic Clinic during the examinations which preceeded diagnosis and assessment. The

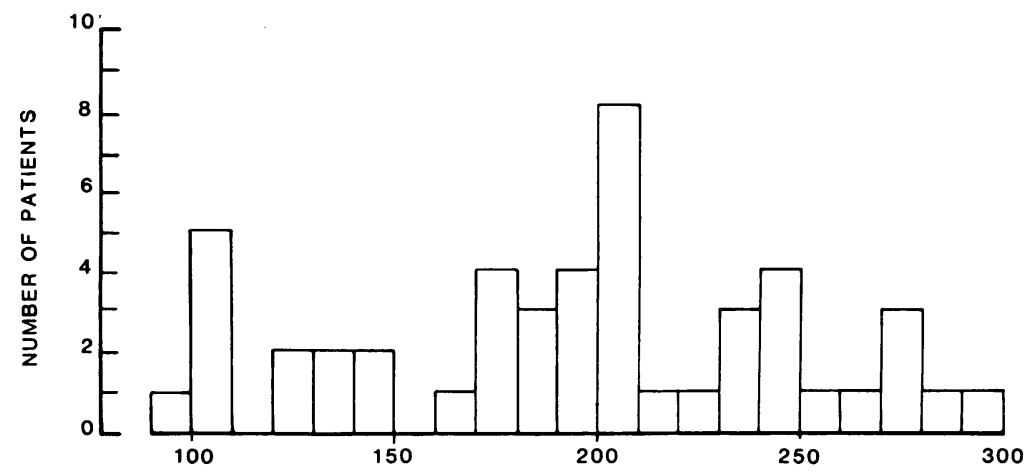

Fig. 1 EOGs in patients of this series. Note the number with indices below 180. 
Fig. 2 Relationship of visual acuity to EOG value. The open symbol indicates that the result is from a patient with Stargardt's disease. There is no relationship between loss in acuity and the EOG value.

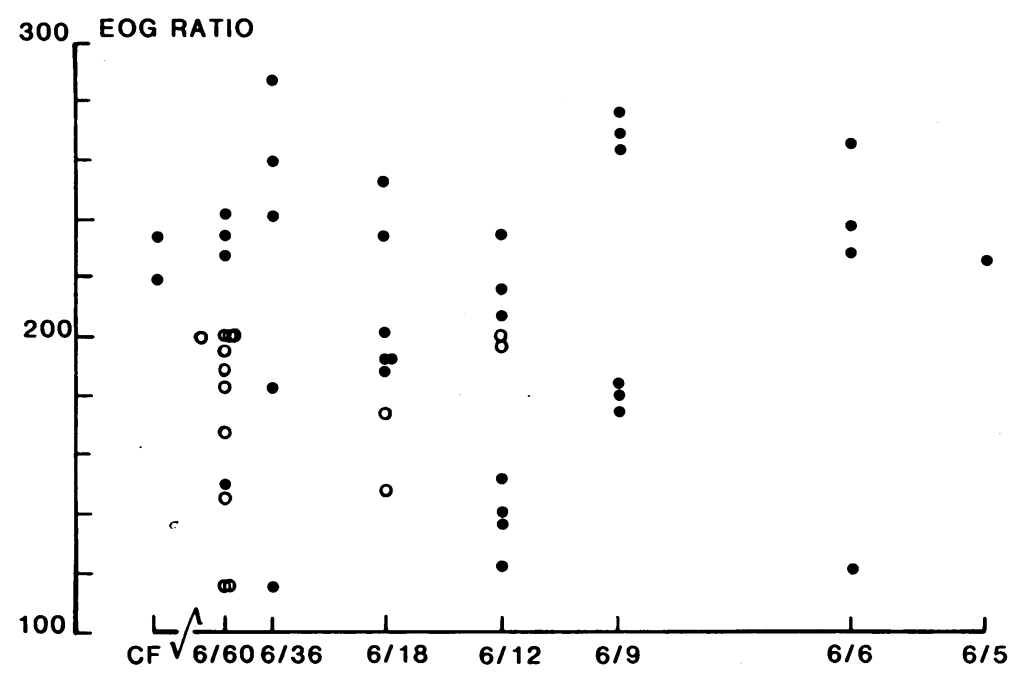

VISUAL ACUITY relationship of the degree of visual impairment to various electrophysiological tests was determined, and the correlations determined between the various abnormalities found in the different electrophysiological tests.

\section{ELECTROCULOGRAMS}

The EOG was recorded in 29 patients. It was normal in 15 and abnormal in 14, who either suffered from juvenile macular dystrophy ${ }^{2}$ or who had a very severe maculopathy. In two cases one eye only was abnormal. In one case of non-specific maculopathy the findings were: right eye $190 \%$, left eye $170 \%$; and in a patient suffering from Stargardt's disease: $\mathbf{R}$ $180 \%$, L $205 \%$. In addition there were two cases of uniocular disease, a man with a left macular solar burn which did not affect the EOG (R $230 \%$, L $225 \%$ ), and a woman with macular degeneration of the right eye only ( $R 243 \%$, L $275 \%$ ). The average value for the EOG index in this study is $187 \%$, SD $52 \%, n=58$, which is below normal value for our population mean. The distribution of results is shown in Fig. 1. It can be seen that there are a number of abnormal results, and a suggestion of a trimodal distribution. There is no relationship between loss of visual acuity and reduction of the EOG ratio (Fig. 2). The correlation coefficient is $-0 \cdot 19$, and the slope of the line is -0.35 (not significant).

\section{GANZFELD ELECTRORETINOGRAM}

Results in individual patients. The Ganzfeld ERG was recorded in 36 patients, and in 11 of them was entirely normal by the strictest criteria. However, in 25 some abnormality was seen, consisting of minor borderline increases in the time to peak of the responses and in some cases reduction in amplitude of the b wave. In some isolated responses the reduction was below 2 standard deviations from the normal mean, though this was never the case for all the ERGs recorded. Such abnormalities were seen in the scotopic responses of three patients and in the cone components in eight, while in 14 both red and cone signals showed abnormalities. Comparisons of results in individual patients are instructive. The patient most affected gives the results shown in Fig. 3, left-hand column, while the centre column shows the results from a patient with uniocular disease. These records were chosen for illustration because the degree of ERG abnormality places the eye in the centre of the distribution. The responses from the fellow eye, which are of course normal and are of average amplitude, are shown in the right-hand column. The differences between the ERGs in the two eyes are not at all obvious. Fig. 4 shows the pattern ERGs obtained in this patient, and it can be seen that the difference between the two is gross, for in the affected eye with reduced visual acuity the response is one-third of that in the fellow eye.

A subset of 14 patients (see 'Material and methods') were examined in more detail. Of these, six were found to have no ERG abnormality. In only three was the amplitude reduced and the time to peak of the $b$ wave increased in both eyes. In one patient with typical Stargardt's disease the ERG abnormality was monocular. In three, time to peak of the $b$ wave was prolonged (one with Stargardt's disease, one with vitelliruptive degeneration, and one who had an atypical cone dystrophy). In one patient the ERG 
A
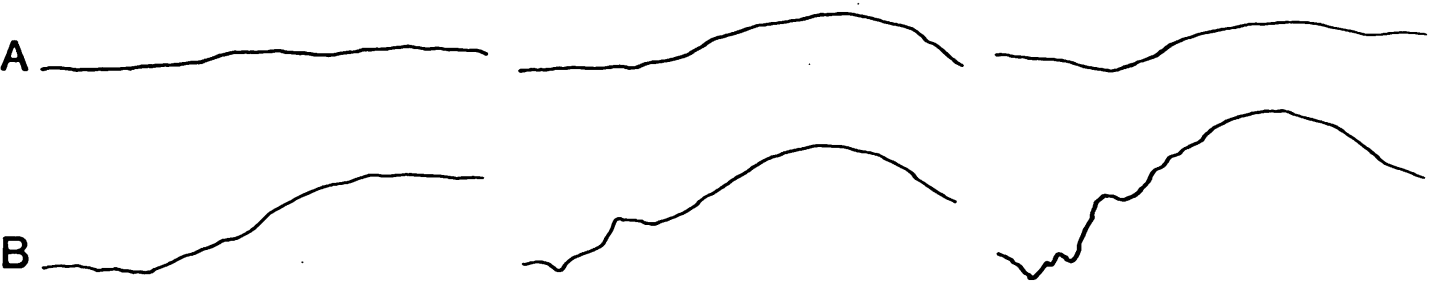

C
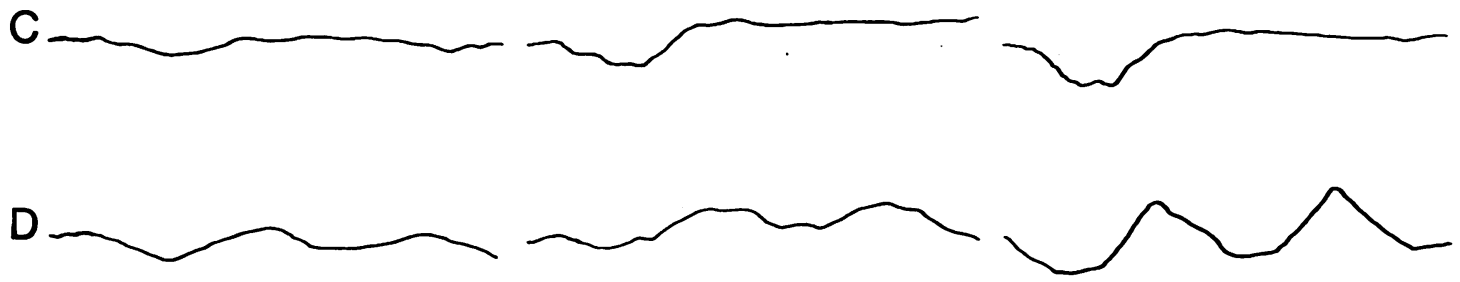
A : $\operatorname{dim}$ blue ( $0.3 \mathrm{q} / \mathrm{rod})$
B : red light ( maximum )
C : white light ( maximum )
D : $30 \mathrm{~Hz}$ flicker

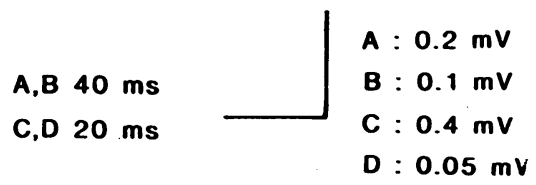

Fig. 3 Ganzfeld ERGs in maculopathy. The stimulus conditions for row A were blue light, c. 0.3 quantalrod/flash; row B maximum red light; row C maximum white light; row $D 30 \mathrm{~Hz}$ flicker. The ERGs in the left column are from the patient with the smallest and most affected ERGs. Note the small size of the scotopic b wave, absence of an early cone complex (red and white light, and the poor flicker responses. The centre and right-hand columns are from a patient with a right uniocular lesion, which reduced the ERG to the centre of the distribution. The cone components are present but reduced. Compare this with the right-hand column, which shows the results from normal fellow eye.

amplitude was reduced in one eye and the time to peak of the $b$ wave increased in both. By the normalising system described in 'Material and

$\mathbf{R}$
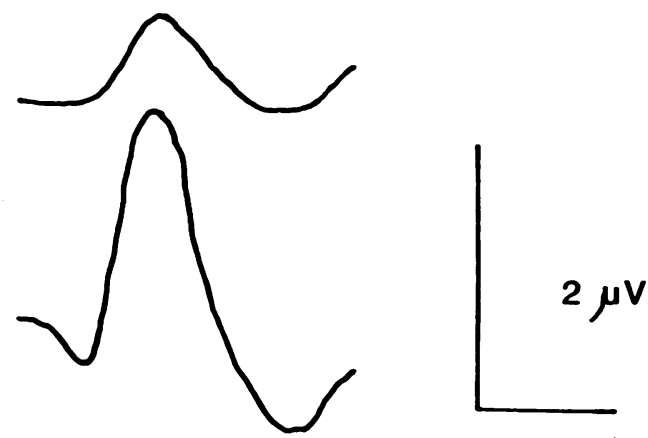

$50 \mathrm{~ms}$

Fig. 4 Pattern ERGs from patient with uniocular macular degeneration (cf. Fig. 3). Note the great difference between the PERGs in the two eyes. methods' the average response amplitude was 0.735 of the mean normal value. The probability of this occurring by chance in any one trial is $0 \cdot 22$, but, when averaged over all trials, $p=10^{-7}$. Analysis of the time to peak of the response also indicates an abnormality. For all trials the average increase in the time to peak was 0.058 of the mean normal value. Although in a single trial such findings are not significant, when the delay is common for all trials of all patients the probability that it occurs by chance is $10^{-6}$. These statistics also indicate that the patients with Stargardt's disease have smaller b waves than the remainder of the patients (Table 2).

The results were analysed to see if any particular form of stimulus was more effective in distinguishing between patients and normal persons. Reductions in amplitude of the $b$ waves were produced by blue light (and also the rod response evoked by red light). The response to maximal white light was also reduced.

The implicit time of the $b$ wave was increased significantly for dim blue light (and the rod com- 
Table 2 Group mean ERGs of maculopathy patients which are highly significantly different from normal

\begin{tabular}{|c|c|c|c|c|c|}
\hline \multirow{2}{*}{$\begin{array}{l}\text { Stimulus* } \\
\text { Protocol number }\end{array}$} & \multirow[b]{2}{*}{ Description } & \multicolumn{2}{|c|}{$\begin{array}{l}\text { Amplitude } \\
\mu \mathrm{V}\end{array}$} & \multicolumn{2}{|c|}{$\begin{array}{l}\text { Implicit time } \\
\mathrm{ms}\end{array}$} \\
\hline & & a Wave & b Wave & a Wave & b Wave \\
\hline \multicolumn{6}{|c|}{ Stargardt's disease $(n=8)$} \\
\hline \multirow[t]{2}{*}{$\begin{array}{l}4 \\
7\end{array}$} & Dim blue flashes & & $\begin{array}{r}29 \pm 17 \\
120 \pm 42\end{array}$ & & \\
\hline & Red flashes: & & & & \\
\hline 17 & $\begin{array}{l}\text { cone ERG } \\
\text { rod ERG }\end{array}$ & $\begin{array}{l}16 \pm 8 \\
11 \pm 5\end{array}$ & $45 \pm 23$ & & \\
\hline 18 & $\begin{array}{c}\text { rod ERG } \\
\text { White flashes }\end{array}$ & $16 \pm 5$ & & & \\
\hline 19 & & $61 \pm 43$ & & $26 \pm 1 \cdot 5$ & \\
\hline 20 & $\cdot$ & & & $23 \pm 1 \cdot 1$ & $49 \pm 2$ \\
\hline \multirow[t]{2}{*}{21} & & & $190 \pm 56$ & & \\
\hline & Flicker & & & & $34+10$ \\
\hline \multicolumn{6}{|l|}{ Others $(n=6)$} \\
\hline & Bright bluc flashes & & & & \\
\hline \multirow{2}{*}{11} & & & & $33 \pm 2 \cdot 1$ & $76 \pm 10$ \\
\hline & $\begin{array}{r}\text { Red flashes } \\
\text { rod ERG }\end{array}$ & $17 \pm 4 \cdot 5$ & & & \\
\hline \multirow[t]{2}{*}{18} & & $15 \pm 4$ & & & $115 \pm 10$ \\
\hline & White flashes & & & & \\
\hline 19 & & & & $25 \pm 1 \cdot 1$ & $50 \pm 4 \cdot 6$ \\
\hline \multirow[t]{2}{*}{20} & & & & $23 \pm 1 \cdot 3$ & $48 \pm 2 \cdot 5$ \\
\hline & Flicker & & & & \\
\hline 31 & & & & & $31 \pm 3$ \\
\hline
\end{tabular}

*Numbers in parentheses refer to the protocol described in the appendix of Arden et al. ${ }^{10}$

ponent of red light flashes), and for white light. The a wave peak time was increased for white flashes (cone activity). These results are shown in Table 2.

\section{PATTERN ERGs}

Normal values. Pattern ERGs were recorded in a number of normal subjects. Some were employees of the hospital, others were relatives of patients who came to the hospital. In all of them the responses to $1^{\circ}$ and $8^{\circ}$ squares were recorded. The former produced a pattern response and the latter, a response to change of illumination of small focal retinal regions
Fig. 5 Normal PERGs (solid circles) and FERGs (open circles) as a function of age.

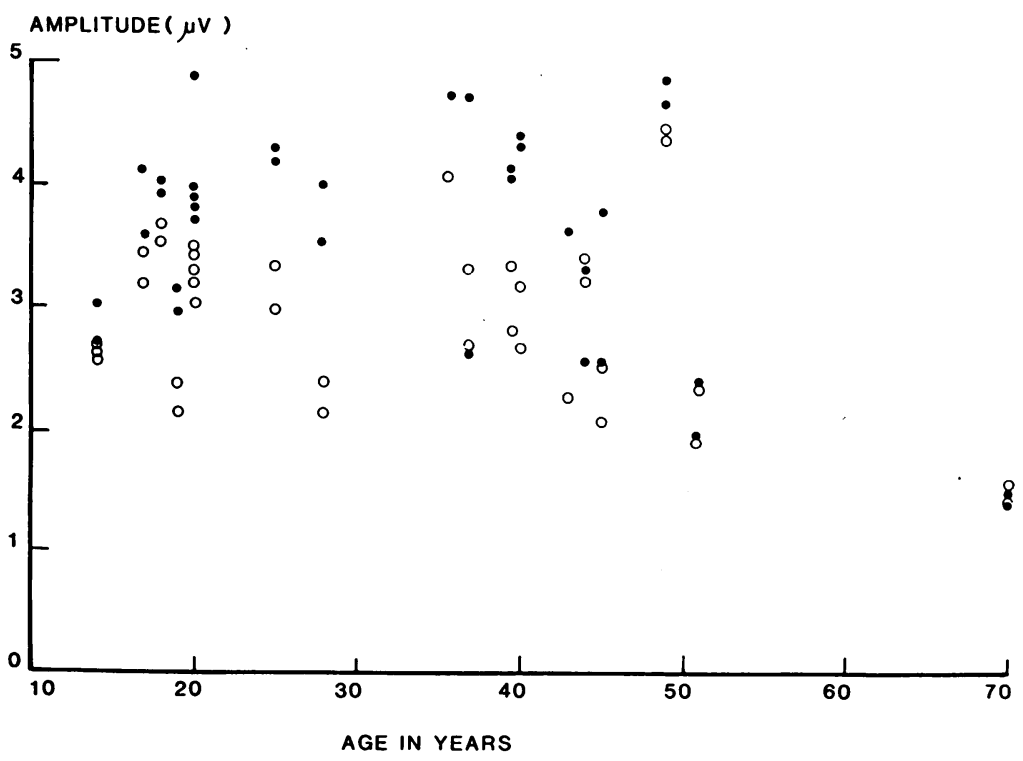


Table 3 Pattern and focal ERGs. Comparison between patients and normal persons

\begin{tabular}{|c|c|c|c|}
\hline \multirow[t]{2}{*}{ Group } & FERG & PERG & PERG/FERG (ratio) \\
\hline & Mean SEM & Mean SEM & Mean SEM \\
\hline $\begin{array}{l}\text { Normals } \\
\text { Patients }\end{array}$ & $\begin{array}{l}3 \cdot 13 \pm 0 \cdot 14 \\
0 \cdot 82 \pm 0 \cdot 1\end{array}$ & $\begin{array}{l}3 \cdot 73 \pm 0.16 \\
0.61 \pm 0.08\end{array}$ & $\begin{array}{l}0.86 \pm 0.02 \\
1.66 \pm 0.3\end{array}$ \\
\hline
\end{tabular}

All differences between patients and normal persons and between PERGs and FERGs very highly significant, $\mathrm{p}<\mathbf{0 . 0 1}$.

(FERG). ${ }^{6714}$ From the age of 9 to 75 years it appears that the mean value of the normal PERG is $3.7 \mu \mathrm{V}$ under the circumstances of our tests (Fig. 5).

The limit of error of the mean (2SEM) is $\pm 0.3 \mu \mathrm{V}$, while the lower limit of normal of an individual result is $2 \cdot 7 \mu \mathrm{V}$. PERGs were recorded in 40 patients with maculopathies. Only three gave a normal PERG recording (amplitude mean $=3.03 \mu \mathrm{V}$ ) in both eyes. Two of these patients had vitelliform maculopathy, but the retina overlying the deposited material was still intact, and vision was preserved. One had a solar retinopathy. Of the remaining 37 two suffered from monocular eye conditions, and the PERG from the fellow eyes was found to be within the normal limits. For the remaining eyes the average amplitude was $0.87 \mu \mathrm{V}, \operatorname{SEM} 0.7$, which is less than one-quarter of the normal value (Table 3 ). Thus the abnormality in the PERG is very obvious, unlike that in the Ganzfeld ERG.

FERG and PERG. Focal and pattern ERGs were obtained in normal persons and the results compared with those from a series of patients with Stargardt's disease, who formed a homogeneous group. The aim was to determine whether the use of the patterned stimulus resulted in a greater reduction in response amplitude than did one which evoked luminance responses. In the normal population the FERG is smaller than the PERG (Fig. 3), so the ratio of the response amplitudes is less than unity. The average value is $0 \cdot 86$, with a range $0 \cdot 61-1 \cdot 18$. In only five cases was the result more than unity. In the patients with Stargardt's disease the PERGs and FERGs were very small, and therefore there are difficulties in assessing the ratio. If all the voltages are summed, the ratio of the averaged FERG to the averaged.PERG is 1.66 , which is considerably different from the normal. However, in some cases the response was judged to be absent or smaller than the electrical noise. In such an individual the ratio could be almost zero or very large indeed. In order to obtain an estimate of the variability of the FERG/PERG ratio we eliminated all those results (11 eyes) where satisfactory measurements could not be made. The average of the ratios and the standard deviation were then calculated. In these patients the mean was also
$1 \cdot 66$, and the value is significantly different from normal. It appears that the PERG was reduced to a greater extent than the FERG in these patients.

\section{CORRELATIONS BETWEEN TESTS}

Visual acuity. Losses in visual acuity were unrelated to the abnormality in the EOG or the ERG. When we divided patients into two groups, those with normal ERGs and those without, the average visual acuity was similar in the two. If the grouping was restricted to presence of abnormalities in the cone ERG, once again the visual acuity of the latter group was (on average) the same as the former. However, five patients with normal cone ERGs had a vision of $6 / 6$ or better, and none of those with ERG cone abnormalities had vision of $6 / 6$ or better. The relationship between the reduction in PERG amplitude and the reduction in visual acuity is shown in Fig. 6. The relationship is highly significant $(p<0 \cdot 001)$.

\section{ELECTRORETINOGRAMS}

We calculated the degree of ERG abnormality by counting the number of abnormal ERGs found in each patient (all of whom were investigated by our standard protocol). The number of failures varied from the averaged of 14 to 1 per eye (the maximum

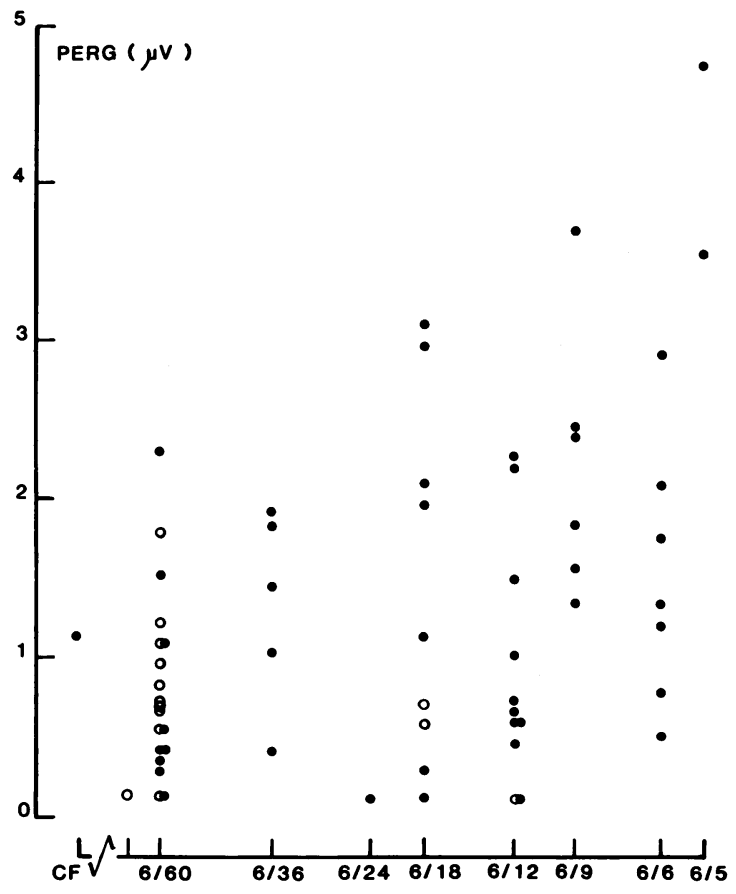

VISUAL ACUITY

Fig. 6 Relationship between PERG amplitude and visual acuity. 
number possible is 22 per eye). We split the patients with abnormalities into two groups. Twelve with the most obvious Ganzfeld ERG changes had a mean PERG of $1.08 \mu \mathrm{V}$, SEM 0.7, and 13 with fewer or lesser reductions had a mean PERG of $0.75 \mu \mathrm{V}, \mathrm{SEM}$ 0.63 .

\section{Discussion}

These results show that in patients with maculopathies the pattern ERG is abnormal in all but the mildest cases. This finding is in contrast with the results with the Ganzfeld ERG, where in many cases the ERG was within normal limits, and reductions in amplitude or increases in peak time were not usually seen in more than a fraction of the records obtained. Such a finding is not unexpected in some cases of maculopathy, because, as can be seen ophthalmoscopically, the condition is usually localised around the macula, and peripheral regions of retina may generate normal responses. In other cases, where the EOG is abnormal, there must be large areas of abnormal pigment epithelium, but it could be argued that the retina continued to function. The region stimulated by our TV monitor is larger than the fovea and approaches that seen in a fundus photograph. The extremely small size of the PERGs seen in conditions which are apparently well localised implies that retinal disturbances may extend beyond the limit of that visible in fundus angiography. In some cases abnormalities on the PERG are evident when minimal vascular leakage can be seen. The sensitivity of the PERG in detecting retinal abnormality is very high. It is satisfactory that it is possible to show a correlation between loss of visual acuity and reduction in PERG amplitude. The significance is reduced by the fact that even very slight reductions in acuity can lead to gross reductions in the PERG. The only exceptions are where the condition is extremely localised (solar burns) or where the retinal function is not lost (unruptured vitelliform maculopathy). ${ }^{5}$ It is interesting that in Stargardt's disease the focal ERG is not reduced to the same extent as the PERG. Since the condition originates in the photoreceptorpigment epithelium complex, both responses must be affected. The additional reduction in the PERG could be accounted for in several ways. For example, the optical image could be degraded by the deposits.
There is no reason to suppose that the differentiation between the FERG and PERG in Stargardt's disease indicates that the two responses are generated in different retinal layers or by different retinal processes.

We wish to thank our colleagues in Moorfields Eye Hospital and from other eye departments for allowing their patients to participate in this study. The work was supported by grants from the Medical Research Council and the Wellcome Trust.

\section{References}

1 Arden GB, Bankes JLK. Foveal electroretinograms as a clinical test. Br J Ophthalmol 1980; 50: 740.

2 Jacobson SJ, Sandberg MA, Effron MH, Berson EL. Foveal cone electroretinograms in strabismic amblyopia. Comparison with juvenile macular degeneration, macular scars and optic atrophy. Trans Ophthalmol Soc UK 1979; 99: 353-6.

3 Sandberg MA, Jacobson SG, Berson EL. Foveal cone electroretinograms in retinitis pigmentosa and juvenile macular dystrophy. Am J Ophthalmol 1980; 88: 702-7.

4 Johnson EP, Riggs LA, Schick AML. Photopic retinal potentials cvoked by phase alternation of a barred pattern. In: Clinical clectroretinography. Vision Res 1966; 6 (suppl): 75-81.

5 Arden GB, Carter RM, Hogg CR, Siegel IM, Margolis S. A new gold foil electrode: extending the horizons of clinical electroretinography. Invest Ophthalmol Visual Sci 1979; 18: 421-6.

6 Arden GB, Vaegan, Hogg CR, Powell DJ, Carter RM. Pattern ERGs are abnormal in many amblyopes. Trans Ophthalmol Soc UK 1980; 100: 453-60.

7 Arden GB, Vaegan, Hogg CR. Clinical and experimental evidence that the pattern electroretinogram (PERG) is generated in more proximal retinal layers than the focal electroretinogram (FERG). Ann NY Acad Sci 1982; 388: 580-601.

8 Bobak P, Bodis-Wollner I, Harnois C, et al. Pattern electroretinograms and visual evoked potentials in glaucoma and multiple sclerosis. Am J Ophthalmol 1983; 96: 72-83.

9 Wanger P, Person HS. Pattern reversal electroretinograms in unilateral glaucoma. Invest Ophthalmol Visual Sci 1983; 24: 749-53.

10 Arden GB, Carter RM, Hogg CR, et al. A modified ERG technique and the results obtained in $X$-linked retinitis pigmentosa. Br J Ophthalmol 1983; 67: 419-30.

11 Arden GB, Vaegan. Electroretinograms evoked in man by local uniform or pattern stimulation. J Physiol (Lond) 1983; 341: 85-104.

12 Arden GB, Faulkner D, Mair C. A versatile television pattern generator for visual evoked potentials. In: Desmedt JE, ed. Visual evoked potentials in man: new developments. Oxford: Clarendon Press, 1977: 90-109.

13 Arden GB, Barrada A, Kelsey JH. New clinical test of retinal function based upon the standing potential of the eye. $B r J$ Ophthalmol 1962; 46: 449-67.

14 Korth M. Pattern evoked responses and luminance evoked responses in the human electroretinogram. J Physiol (Lond) 1983; 337: 451-69. 